\title{
Phénomènes de ruptures dans Verbo d'Eduardo Chapero-Jackson : l'hybridation comme acte de résistance
}

Université Lumière-Lyon 2

Silvina Benevent Gonzalez

\section{OpenEdition}

Journals

Édition électronique

URL : https://journals.openedition.org/cher/3949

DOI : 10.4000/cher.3949

ISSN : 2803-5992

Éditeur

Presses universitaires de Strasbourg

Édition imprimée

Date de publication : 1 décembre 2015

Pagination : $241-248$

ISBN : 978-2-86820-913-9

ISSN : $1968-035 X$

\section{Référence électronique}

Silvina Benevent Gonzalez, «Phénomènes de ruptures dans Verbo d'Eduardo Chapero-Jackson

I'hybridation comme acte de résistance », reCHERches [En ligne], 15 | 2015, mis en ligne le 01 décembre 2021, consulté le 22 décembre 2021. URL : http://journals.openedition.org/cher/3949 ; DOl : https://doi.org/10.4000/cher.3949

\section{cc) (†) (2)}

$\mathrm{Ce}(\mathrm{tte})$ œuvre est mise à disposition selon les termes de la Licence Creative Commons Attribution Pas d'Utilisation Commerciale - Partage dans les Mêmes Conditions 4.0 International. 


\title{
Phénomènes de ruptures dans Verbo d'Eduardo Chapero-Jackson : I'hybridation comme acte de résistance
}

\author{
Silvina Benevent González \\ Université Lumière-Lyon 2
}

$F_{\mathrm{d} e}^{\mathrm{il}}$ lm de fiction, Verbo (2011) n’en est pas moins ancré dans la réalité sociale de l'Espagne contemporaine. Or, ce film est considéré par certains comme une œuvre présomptueuse voire arrogante ${ }^{1}$ et par d'autres comme un film pur, audacieux et dissident ${ }^{2}$ par son traitement fantastique et sa vision stylisée. Un tel antagonisme de jugements invite d'emblée à s'interroger sur la nature, la vision et les processus mis en action dans cette œuvre indisciplinée et récompensée à plusieurs reprises. Il peut sembler surprenant qu'Eduardo Chapero-Jackson qui a acquis une solide réputation en réalisant jusqu'à alors des courts-métrages, délaisse, à première vue, dans son premier long-métrage, une réflexion sociale autour des grands maux de la société actuelle en se confrontant à la jeunesse aux poncifs persistants.

Il s'agit pourtant bien encore d'un récit dramatique qui retient surtout l'exposition d'une série de crises vécues par une adolescente timide et solitaire: Sara. En proie à un mal-être existentiel quelque peu commun aux jeunes gens de son âge, elle se verra attirée par l'anéantissement de soi et devra prendre conscience du dilemme qui s'offre à elle: mourir ou s'affirmer et s'affermir en tant qu'individu.

1 «Una primera película soberbia, o sea, altiva, arrogante [...] que tanto puede ser tomada entre risas como provocar una llantina y que, eso sí, preludia a un cineasta tenso e intenso», (Rodríguez Marchante 2011).

2 «Qué sencillo resulta apedrear con vil cinismo esta cinta pura, atrevida y disidente, de tan audaz fragilidad como esa voz única, sin miedo al ridículo, casi ahogada en la manada, y cuyo mensaje, dirigido a quien jamás lo escuchó antes, encima, es verdad.» (Trashorras 2011). 
Toutefois, la transformation de la jeune fille débute par une quête de l'invisible, telle une Alice des temps modernes, au sein d'une urbanité nouvelle mais monstrueuse, creuset d'une certaine idée de crise. Et ce sera tout l'enjeu de notre première partie. Nous analyserons ensuite de quelle manière le réalisateur traduit, dans une prise de risque audacieuse, la fusion du monde visible et du monde invisible par un mélange vraisemblable des genres entre le graffiti, la science-fiction, le film d'animation, les jeux vidéo et le film fantastique. Enfin il y a lieu de penser que dans la banalité du quotidien, la rupture engendrée par la crise inclut bel et bien l'altération d'un ordre ancien et la naissance d'une forme nouvelle.

S'il nous a semblé significatif d'aborder ce film selon la thématique de la crise en tant que changement, transformation et rupture, nous nous proposons de l'examiner aussi selon un axe réflexif qui démêle l'épineuse question de la modernité, d’abord à partir des travaux de Néstor García Canclini, puis de ceux de Miguel Benasayag. Entre altération du paysage urbain, volonté de rupture des personnages et transmutation de la parole, affleure peut-être aussi une capacité créatrice née d'un processus de revendication en écho à l'affirmation de Miguel Benasayag pour qui «Résister, c'est créer $»^{3}$. Il s'agira de voir en quoi la capacité créatrice et une esthétique saisissante née d'hybridations peuvent porter en elles un appel à la résistance quand la notion de crise intègre aussi le signe d'une transformation multidimensionnelle en profondeur.

\section{Mutations et mobilités dans l'espace urbain}

À l’origine, Eduardo Chapero-Jackson est un fervent défenseur du genre court dont il soutient la légitimité et l'essence poétique. Poète de la vie quotidienne ${ }^{4}$, il a exprimé dans chacune de ses œuvres une vision personnelle de thèmes modernes et préoccupants de la société actuelle. Contracuerpo en 2005 traite de l'anorexie et de l'enfer de la mode: il y dépeint l'obsession d'une jeune fille à glisser son corps amaigri dans un mannequin de vitrine jusquà se liquéfier littéralement. Alumbramiento (primé à Venise) en 2007 mesure les différentes façons d'appréhender la mort au sein d'une famille réunie la nuit de l'agonie d'une vieille dame. En 2008, The end, projeté à l'exposition universelle de Saragosse (sur le thème de l'eau et du développement durable), dresse un tableau effrayant des conséquences du manque d'eau dans le monde. Ses trois courts-métrages réunis dans une trilogie A contraluz lui ont valu plus d'une centaine de prix.

Poursuivant son questionnement sur les grands changements sociaux de l'Espagne des dernières années, il porte dans Verbo, un regard acéré sur les fractures imposées à l'individu à l'âge où il est le plus fragile, à savoir l'adolescence. Entre un père architecte peu présent et une mère dépassée, Sara, à l'aube de ses

3 D’après l'hypothèse posée par Gilles Deleuze, cité par (Aubenas et Benasayag 2002: 7).

4 L'aspect poétique est véritablement une préoccupation du réalisateur comme le montrent nombre de ses interviews. On pourra consulter (Rull 2011) et (Iglesia 2012). 
15 ans, est une jeune fille peu sociable. A priori, cette famille espagnole mène une existence presque banale dans une banlieue anonyme et résidentielle d'une grande ville, Madrid.

Pourtant, le film débute par la vue nocturne d'une autre ville que nous identifions comme étant Tokyo, illuminée par une multitude de lumières et reconnaissable à ses gratte-ciel. Un univers en somme lui aussi caricatural où lêtre humain est pétrifié, ce qui est corroboré par la succession de scènes rapides: trois plans de Tokyo s'enchaînent tels des flashs, juxtaposés à des coups de gong comme des coups de semonce. Mais dans cet espace gigantesque et sans âme, un drame se déroule dans un appartement sombre: un jeune homme, sourd aux appels désespérés de ses parents, s'enferme dans la salle de bain et se suicide.

À cet espace marqué par le tragique, succèdent le titre puis la première image du lieu de vie de Sara: un cimetière de pneus, montagne noire d'objets concentriques. Ils sont les vestiges d'une économie florissante qui donnent la mesure des schémas antérieurs d'un pays aujourd'hui en crise, en proie à un cercle vicieux auquel il ne semble plus pouvoir échapper.

D’emblée, en voix off, Sara, se définit par rapport à son lieu d’existence «Me llamo Sara. Tengo 15 años. Éste es mi mundo. Recorro estas calles como un fantasma». Elle s'identifie donc dans un premier temps comme résidente d'un territoire indéfini mais définissable. Elle est pourtant d’ores et déjà une habitante absente au mouvement limité dans la torpeur d'une ville-dortoir de la banlieue madrilène.

Aussi se contente-t-elle d'observer un graffiti qui incite à l'action, à la réflexion et à la prise en main individuelle, au pied d'un immeuble en ciment et en briques, matériaux traditionnels de la construction moderne en Espagne; noublions pas que son père est architecte.

Dans ces marques reconnaissables qui «plantent» le décor, c'est le cadre réel qui s'immisce, celui des villes nouvelles nées de la dite «bulle» immobilière. Comme conséquence de la crise qui frappe de plein fouet l'Espagne, ces villes périphériques aux édifices désertés en attente d'habitants sont effectivement surnommées "ciudades fantasmas». On peut dès lors affirmer que les repères spatiaux sont ici fondamentaux dans la mesure où ils incarnent des stratégies pour préfigurer les grands changements sociaux, économiques et politiques qui pèsent sur une société espagnole en proie au désenchantement et à la rupture.

Dans la ville monstrueuse, car dépouillée de ses fonctions de vie et d'échange, l'adolescente est ainsi victime du manque de perspectives de son présent «me siento enjaulada» confiera-t-elle. Pire encore, le gel de l'espace conditionne son mutisme: elle se sent rejetée dans un espace lui-même marginalisé. Elle est irresponsable pour les uns, folle pour les autres. Comme le jeune Japonais en ouverture, elle finit donc par envisager et organiser son propre suicide en se jetant par la fenêtre de sa chambre. C'est par ce biais que le lien se crée entre les deux villes des premières séquences. 
Cette attirance de Sara pour le vide et l'oubli de soi s'accompagne auparavant d'une recherche de l'ailleurs. Un espace autre qu'elle entrevoit et devine derrière les graffitis qui fleurissent de-ci de-là signés par Liriko. La relation duelle de la ville versus l'imaginaire s'instaure donc à travers une relation spéculaire qui marque une trêve dans le récit premier, reflet d'une réalité vérifiable. Anticipant son suicide, Sara dépose au préalable au point d'impact de sa chute plusieurs plaques de verre pour recevoir son corps défenestré et lui permettre de passer de l'autre côté, transition du corps fictif. Elle répond ainsi à lıappel de Liriko écrit sur un miroir: "Adéntrate en mí».

Par une logique de collages, le film met en jeu des horizons modernes et vise alors à relégitimer un espace marginalisé. Tout est brusquement mobile, les espaces ne sont plus clos. Par conséquent, la crise et la rupture qui s'ensuit sont synonymes de changements voire de désengagement face au modèle initial. Et le désengagement s'opère par le biais de la dialectique des genres.

\section{Dialectique des genres}

Finalement, l'inadéquation de son être avec le monde dans lequel elle vit et avec les exigences qui lui sont imposées provoque chez Sara le sentiment dêtre déphasée. Un sentiment qui n'est pas étranger à son état d'adolescente, contraint entre l'enfant qu'elle quitte et l'adulte qu'elle devient, ce qui est source de conflit intérieur. Dans un souci maximaliste de déconstruction, l'espace réaliste explose, disions-nous, et Sara s'affranchit de ses limites jusqu’à un espace invisible, celui du champ des possibles. Dans ce monde d'illusions auquel les autres ne croient pas $^{5}$, elle est guidée par le graphiste Liriko qui l'exhorte à entrer en action. Sara découvre notamment le sens profond de la vie dans la lecture d'une oeuvre emblématique, Don Quichotte. Elle s'identifie peu à peu au héros cervantin, persuadé lui aussi que la vertu de lêtre humain est de rendre à l'humanité son innocence antérieure et de rendre possible ce qui semblait auparavant impossible.

Sollicitée par les messages mystérieux de Liriko, Sara elle-même se lance dans une aventure quichottesque: sauver une maison, symbole d'un ordre ancien, de la démolition à laquelle des promoteurs avides de profits la vouent sans vergogne. Ici, point de géants aux allures de moulins. Pourtant, les immeubles sont une véritable barrière aux illusions et utopies adolescentes. En ce sens aussi, l'espace est étouffant et toutes les ouvertures (les arcades, les cours intérieures et les fenêtres) agissent comme des signes d'encerclement. Les façades de briques retiennent en somme les individus en suspens, pris en étau entre deux histoires, deux destins, et donnent la mesure de la tâche qui s'offre à l'adolescence.

Dans un jeu similaire de confusion des niveaux narratifs, le réalisateur fait feu de tout bois et bouleverse des genres bien établis afin de marquer la porosité des frontières et traduire la fusion d'espaces antithétiques. Le film se présente,

5 Elle est assaillie par tous: sa mère lui dit «Tienes que ser más realista», son ami lui écrit «Céntrate» et son professeur la rappelle à l'ordre, «¿En qué mundo estás?». 
en effet, comme une véritable fabrique intertextuelle. C'est ainsi que l'espace diégétique introduit le monde enfantin à travers les réminiscences des contes. Et si Sara scrute derrière un miroir, comme Alice de l'autre côté du miroir, elle cherche bel et bien à s'introduire dans un monde parallèle: «tiene que haber algo ». Plus tard, elle sème des pièces de puzzle dans l'appartement et dirige ainsi sa mère jusqu'à son lit, lui indiquant tel le Petit Poucet le lieu de l'enfance et de l'innocence et lui suggère qu'elles peuvent encore se retrouver. Une fois la frontière franchie, Verbo n'a de cesse de brouiller les cadres génériques de rigueur. D'une part, l'accélération du mouvement et l'acceptation de l'action par Sara se jouent par l'intrusion du clip-vidéo, marqué par la musique et la présence même du rappeur Nacht. Plus encore, comme dans les films d'animation, Sara est capable de se transformer en hérö̈ne d'animation, de traverser les murs et d'entraîner encore ses amis vers une autre dimension. Verbo se situe parfois aussi au croisement du film de fiction et de l'esthétique du jeu vidéo. Donnons comme exemple la scène où Sara chausse des patins et se déplace à vive allure sur des rails aériens et multicolores.

Le champ des possibles est d'autre part élargi par des références diverses: Sara livre une bataille verbale contre sa propre volonté suicidaire. C'est le combat, aux allures de science-fiction, de l'obscurité contre la lumière dont l'issue marque l'envie de vivre de l'héroïne. Quitte à résister à l'attrait de l'anéantissement, Sara affronte aussi une morte-vivante dans son appartement dévasté et noirci. La claire fusion de cette scène avec le genre fantastique est tout aussi efficace. Il est frappant de voir que, finalement, le réalisateur en appelle aussi à des œuvres emblématiques du cinéma ayant en commun une certaine idée du conflit: Matrix d'Andy et Lana Wachowski, Star Wars de George Lucas ou encore Subway de Luc Besson.

Que pouvons-nous retenir de tous ces exemples? Sans doute que les changements de perspectives incessants appuient le pouvoir émotionnel des images mais pas seulement. Il y a une recherche constante de rendre diaphane ce qui était opaque dans un premier temps. Lêtre n'a de sens que dans la dimension plurielle, dans toute la complexité de la substance. Le réalisateur cherche à pénétrer la subjectivité en écrivant une histoire certes naïve mais qui franchit les frontières perméables et mouvantes de l'imaginaire qui repose aussi sur des processus historiques, culturels et générationnels. D'où un personnage protéiforme.

Qui plus est, le caractère exhibitionniste de la parole dans Verbo, participe clairement de cette idée, un comble quand le film met en scène une jeune fille caractérisée par son mutisme. Malgré tout, le mot surgit de l’ombre: «Yo soy el ruido en el silencio » affirme Liriko, le lyrique. Et entre le mot et sa représentation, il y a le graffiti. Contre-culture, le graffiti est une inscription de soi, un marquage identitaire sur les murs de la ville et le signe d'une revendication qui exprime une autre vision du monde entre désenchantement, utopie et espoir. Enfin, il est la possibilité d'un texte à inscrire comme nouveau support d'écriture éphémère et périssable pour le moins marginalisée car déconsidérée, rejetée et 
finalement effacée. Mais son inévitable effritement est paradoxalement sa force de persuasion, sa propre pertinence.

Si dans Verbo, les graffitis sont autant de formes de polyphonie à travers des palimpsestes, ils inscrivent en définitive des dialogues propices à faire naître des liens entre la sphère individuelle et la sphère collective. Ils sont l'empreinte de l'invisible sur le visible puisque l'on découvre l'envers du mur sur lequel susurrer quelques mots suffit à leur donner forme de l'autre côté.

\section{Création et résistance}

Voyons maintenant comment depuis les limbes d'une "pré-mort», Sara, au bord de l'abîme, se sent soudain partie prenante d'un ensemble quand son destin se joue à plusieurs voix, celle de Liriko et de ses acolytes. L'effraction de voix multiples donne littéralement du relief aux messages auparavant simplement apposés sur les murs. D’ailleurs, le lyrisme n’est-il pas une «manière passionnée, poétique, de sentir, de vivre» d'après une acception du petit Robert?

Il résulte de tout ceci que la parole est centrale et ce, par-delà la platitude de la surface. De toute évidence, l'affirmation de soi à travers le mot et le verbe marque la victoire de Sara face à son alter ego transpercé par les éclats de verre de miroir : «Estoy aquí, yo decido, soy mi alma, soy mi cuerpo, yo digo, yo amo, yo elijo, yo lucho, yo siento, yo soy Verbo». En somme, du langage parlé à l'empreinte de la parole sur les murs, il y a une transformation radicale puisque sa parole contient toutes les personnes sans qu'elle renonce à son individualité. Il y a ainsi un dépassement d'une conception manichéenne infertile. Sara qui accepte d'apprendre de ses erreurs, d'aimer et d'en assumer le risque, de se relever après la chute, entre dans un système où le tout est dans chaque partie comme le souligne Michel Benasayag (2012: 126): «si nous voulons connaître l'état d'un système, nous ne pouvons y avoir accès qu'en intériorité, dans chaque multiple qui le compose.» La parole n'a de valeur que si elle est adressée aux autres. Sara devient oratrice devant ses camarades le temps du chapitre L de Don Quichotte qu'elle a transformé et adapté à son histoire. Elle montre également toute la difficulté à sortir du champ pour/contre pour vivre avec ses paradoxes intrinsèques, dans cette leçon quelle donne à son professeur sur l'œuvre de Miguel Cervantes:

Sepa vuestra merced que no es consciente de la sabiduría que este libro encierra y para qué nos sirve y en qué nos concierne [...] me miráis como una loca pero lúcida estoy. Tan sólo no tapo mi boca.

Loin d'offrir un système de représentation auquel le spectateur viendrait illusoirement s'identifier, le procédé propose un champ à l'énonciation, aux potentialités de l'acte créatif dans un système qui joue à la fois de la permutation des personnes et du recoupement des membres de l'ensemble.

Eduardo Chapero-Jackson lui-même renonce à un genre, à un langage usuel du genre donné et manipule une idéologie hybride qui abat les murs entre illusion/réalité, forme/fonction et fantaisie/praxis. C’est dans ce rapport des 
fragments les uns aux autres que se trouve impliqué le rapport «intertextuel», faisant le jeu d'un texte non-clôturé. Le sociologue argentin Néstor García Canclini (2010:2) affirme au sujet des processus d'hybridation que l'hétérogénéité des pratiques

peut se lire comme autant de formes d'une dialectique intense rejoignant la formation et la transformation des formes symboliques par et dans lesquelles se hissent les médiations culturelles de nos sociétés.

Dans la catharsis, Sara s'engage dans une démarche progressive/régressive vers ce qu'on pourrait appeler le point asymptotique de son existence. Elle est de l'ordre de la RE-naissance si l'on considère une succession d'images évocatrices. Dans un rêve, Sara doit couper le cordon qui la relie à sa mère en proie aux douleurs de l'enfantement. Ensuite, elle échappe à l'étreinte d'une petite fille moribonde, clair reflet de cet être qu'elle doit abandonner. Finalement le cri quelle pousse en appelle au premier cri de la naissance. Le rêve s'achève et ses compagnons la libèrent de l'eau dans laquelle ils l'avaient immergée. L'initiation à l'action peut commencer. Et dans le parcours initiatique de Sara, on peut relever trois grands axes. D’abord, le rite passe par la connaissance:

il n'y a pas de connaissance profonde de la situation sans engagement en elle, parce que l'une des conditions fondamentales de la connaissance [...] est la nécessité de l’agir. (Benasayag 2012: 104)

La première épreuve est celle de la prise de conscience: «tu pensamiento».

La réappropriation de soi repose ensuite sur une manière de fluer; de s'affranchir des limites, de sémanciper du carcan initial et d'assumer le conflit comme pour Michel Benasayag: «toute métaphysique de l'affrontement est vaine, car l'affrontement n'est ni vérité ni mensonge. Il n'est que cette dimension que, de temps en temps, nous devons pouvoir assumer» (Benasayag 2012: 114). Il faut se rappeler la deuxième épreuve, celle de l'attitude à assumer. Mais la finalité de toutes ces épreuves est celle de l'action. Laction est bien le principe même des épreuves imposées à Sara qu'elle réussit dans le monde parallèle et qu'elle met ensuite en œuvre dans le mouvement du quotidien:

Si es que has venido a alcanzar el bien que hundido bajo estas negras aguas se haya, muéstranos sin más tu fuerte pecho y arrójate, en su negro liquido mójate una vez y otra hasta que tu vida encuentre algo. Si no es así enójate y sigue, porque si así no lo haces no serás digno de ver el hermoso signo que llevas dentro de ti. ${ }^{6}$

Une fois passées toutes ces épreuves, la conscience et la parole sont siennes.

En fin de compte, la véritable audace de cette œuvre, c'est un assemblage chaotique d'esthétiques diverses qui parvient néanmoins à concilier voire réconcilier la culture moderne et la littérature "patrimoniale» et qui invite à la reconsidérer selon le processus d'hybridation dont parle Nestor García Canclini

6 D'après le chapitre L de El ingenioso hidalgo don Quijote de la Mancha (Cervantes 1971: 473). 
à propos de l'espace latino-américain: l'hybridation entre intertextualité et transculturalité. Elle serait comme une surface non forclose par les relations quelle entretient avec les autres surfaces dans une dépendance et interpénétration de toute surface. «La mise en multiplicité de l'identité est précisément ce qui permet la création du nouveau » écrit Miguel Benasayag (2012:105) comme une manière endogène de dépasser l'état de crise.

Ainsi, la réaction à la crise entre rupture et mutation est affirmation de la liberté. Cette même liberté que revendique Eduardo Chapero-Jackson en désacralisant le genre imposé, qui joue sur la poétique du discours et de l'image pour répondre aux questions et aux angoisses existentielles. Hors des sentiers battus, c'est dans la perspective d'une hybridation dynamique qu'il récidive dans sa dernière œuvre, Los mundos sutiles: un hommage poétique à l’œuvre d'Antonio Machado au sein d'une urbanité à nouveau désolante. À la croisée du passé et du futur, la parole intègre, au sein même de la rupture, l'histoire dans une vaste vision créatrice, la ville de demain. Une vision enfantine et un vœu pieux sans doute mais combien poétique.

\section{Bibliographie}

Aubenas, F. et Benasayag, M., 2002, Résister, c'est créer, Paris, Éditions La Découverte.

Benasayag M. et del Rey, A., 2012, Éloge du conflit, Paris, Éditions La Découverte. Cervantes Saavedra, de, M., 1971, Editorial Magisterio Español, Colección Novelas y Cuentos, Madrid.

García Canclini, N., 2010, Cultures hybrides: stratégies pour entrer et sortir de la modernité, Les Presses de l'Université de Laval.

Rull, C. 2011, «Eduardo Chapero-Jackson, director de Verbo: Los jóvenes están desilusionados», (03.11.2011) http://www.20minutos.es/noticia/1205595/0/ eduardo/chapero-jackson/verbo/ [consulté en avril 2013].

Iglesia, A.M., 2012, «Eduardo Chapero Jackson: En el cine lo poético es peligroso", (15.11.12), Lavanguardia.com, Revista de letras, Espagne, http:// www.revistadeletras.net/eduardo-chapero-jackson-en-el-cine-lo-poetico-espeligroso/ [consulté en avril 2013].

Rodríguez Marchante, E, 2011, Diario ABC, 04/11/2011 http://www.abc.es/ 20111104/cultura-cine/abci-criticas-estrenos-cine-noviembre-201111031325. html, [consulté en février 2013].

Trashorras, A., «Verbo», 2011, Espagne, 04/11/2011, http://www.fotogramas.es/ Peliculas/Verbo/Critica, [consulté en février 2013]. 